\title{
Software ERP
}

\section{ERP Software}

HUERTA-MASCOTTE, Eduardo*† $\uparrow^{1}$, RICO-MORENO, José Luis ${ }^{1}$ ，AGUIRRE-PUENTE，José Alfredo $^{1}$ y MATA-CHÁVEZ, Ruth Ivonne ${ }^{2}$

${ }^{1}$ Ingeniería en Tecnologías de la Información y Comunicación, Universidad Tecnológica del Suroeste de Guanajuato. Carretera Valle-Huanímaro Km 1.2, Valle de Santiago, Gto.CP. 38400

${ }^{2}$ Electrónica y comunicaciones, Universidad de Guanajuato, Av. Universidad S/N, Col Yacatitas, Yuriria, Gto.

ID $1^{\text {er }}$ Autor: Eduardo, Huerta-Mascotte

ID $1^{\mathrm{er}}$ Coautor: José Luis, Rico-Moreno / ORC ID: 0000-0002-1810-6431, Researcher ID Thomson: T-2638-2018

ID $2^{\text {do }}$ Coautor: José Alfredo, Aguirre-Puente

ID $3^{\text {er }}$ Coautor: Ruth Ivonne, Mata-Chávez

DOI: $10.35429 /$ JCS.2019.8.3.18.23

Recibido: 12 de Marzo, 2019; Aceptado 30 de Junio, 2019

\section{Resumen}

En este artículo se presenta información de la implementación de un sistema ERP a la medida. Partiendo del hecho de que una empresa ya cuenta con un sistema ERP en funcionamiento al cual se le pretende añadir un nuevo módulo, con el propósito de rescatar ciertos recursos, para evitar la generación de datos repetidos y obtener un sistema más robusto y seguro. Dicho software

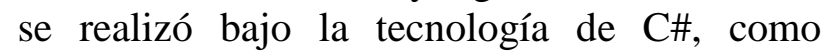
lenguaje de programación y SQL Server, como servidor de base de datos. estableciendo eficiencia y agilidad en el proceso de generación de nóminas.

Sistema ERP, Software, Robusto, Seguro, Optimizar, Recursos

\begin{abstract}
This article presents information on the implementation of a customized ERP system. Based on the fact that a company already has a functioning ERP system to which it is intended to add a new module, in order to rescue certain resources, to avoid the generation of repeated data and obtain a more robust and secure system. This software was made under the technology of $\mathrm{C} \#$, as programming language and SQL Server, as a database server. establishing efficiency and agility in the payroll generation process.
\end{abstract}

ERP System, Software, Robust, Safe, Optimize, Resources

Citación: HUERTA-MASCOTTE, Eduardo, RICO-MORENO, José Luis, AGUIRRE-PUENTE, José Alfredo y MATACHÁVEZ, Ruth Ivonne. Software ERP. Revista de Simulación Computacional. 2019. 3-8: 18-23

\footnotetext{
* Correspondencia al Autor: (ehuerta@utsoe.edu.mx)

$\dagger$ Investigador contribuyendo como primer autor.
} 


\section{Introducción}

En los últimos años ha surgido una revolución dentro de las empresas, principalmente en pequeñas y medianas, puesto que se ha intentado implementar sistemas únicamente existentes en grandes fábricas o comercios. Los sistemas ERP tienen un gran impacto en la manera en que las operaciones son manejadas, además, da una buena primera impresión para cualquier cliente potencial. Especialmente hablando de una empresa con giro en las Telecomunicaciones con impacto, por igual, en el desarrollo de software. En el mercado, se pueden encontrar software que son utilizados para administración de nominas tales como, CONTPAQi Nominas [1], AspelNOI, HR Corporate y Nominasol [2].

En la empresa se contaba con un software ERP funcional, el cual requería de una actualización; para tal efecto, se llevó a cabo la implementación de un módulo adicional, en apoyo al área de recursos humanos. Este software, pretendía mejorar los procesos de las operaciones. Para su desarrollo se utilizó SQL Server, así como el lenguaje de programación C\#. Además, se hizo un análisis de las operaciones que realizaba el sistema ERP actual, así como el uso de la base de datos y la información ya contenida en la misma.

Dicho sistema informático emite nóminas de manera automática, generando un ahorro de tiempo y reducción de errores humanos; reflejando un respaldo de datos que se mantendrán almacenados por el tiempo necesario.

\section{Objetivo}

Ofrecer una solución tecnológica a la problemática actual, satisfaciendo las necesidades del cliente.

\section{Marco teórico}

Toda vez que una empresa en operaciones comienza a realizar pagos al personal que labora para la misma, es necesario calcular la nómina de acuerdo con las normas salariales establecidas por la empresa las cuales deberán, en todo momento apegarse a las leyes vigentes. A continuación, se consideran los puntos más importantes a resaltar.

\section{Nomina}

"Documento de control administrativo, en el cual se consignan las percepciones y deducciones de uno o varios trabajadores que integran una organización en un periodo determinado" [3].

\section{Marco Legal de las Nominas}

Las leyes que rigen a los empleados, así como a los patrones de cualquier trabajo, están designadas y tienen su base legal en el artículo 123 de la Constitución Política de los Estados Unidos Mexicanos. El cual cuenta con algunos apartados de los que, en este estudio se enfoca en los que están relacionados de alguna manera con el tema principal, la nómina.

\section{A) Ley del Instituto Mexicano del Seguro Social [4] \\ B) Ley Federal del Trabajo, articulo 84 [5].}

\section{Tipos de Nomina}

Entre las características que se pueden resaltar en una nómina o tipos de nómina, se tienen:

- $\quad$ Normal: Contiene información sobre las percepciones que recibe el trabajador de forma regular, como lo es el salario y otros ingresos.

- Especial: Contiene percepciones extraordinarias las cuales solo conciernen, de manera más personal, además de sujetas a cambios, por ejemplo, logro de metas, incremento de productividad, pago de utilidades, entre otras.

- Confidencial: Por lo general este tipo de nómina, está destinada para directores, gerentes generales y funcionarios, para este tipo de información, el acceso está restringido.

\section{Cálculo de la nomina}

Para generar la nómina, se fija el salario diario para un empleado, el cual es dictaminado por la empresa. Aquí, se consideran las funciones y responsabilidades que va a desempeñar el trabajador, de forma equitativa en comparación con los demás trabajadores que desempeñen las mismas funciones. 
Dicho esto, se fija el salario diario, para esto se puede consultar en el artículo, el salario mínimo que debe de percibir una persona. Una vez considerado el salario diario a percibir, se realiza el primer cálculo:

\section{Sueldo Bruto $=$ Salario Diario $* 6$ días laborados +1 día por Ley}

Una vez obtenido el Sueldo Bruto del empleado, se tiene que llevar a cabo las deducciones que por ley se marcan, las cuales son:

\section{- $\quad$ Impuesto Sobre la Renta (ISR) el cual es consultado en el portal del SAT \\ Deducción del Seguro Social (IMSS) el cual es consultado en el sistema SUA que proporciona el IMSS [6].}

Es necesario tener actualizados estos números ya que están cambiando constantemente. Un trabajador por horas, no podrá tener el mismo sueldo fijo siempre, debido a esta situación. De igual manera, para el caso del cálculo del ISR, aplica la regla de, al que gana más, se le descuenta más.

\section{Sueldo Neto $=$ Sueldo Bruto $-($ Retención ISR + Retención IMSS)}

Estos dos son los principales factores que deben de estar dentro del desglose de nómina para los empleados, puesto que dentro de la nómina se toman en cuenta todos los beneficios que, por Ley, deben de ser otorgados a un empleado, aunque estos no son otorgados o descontados, cada que se emite una nómina, estos beneficios que también se calculan y afectan el sueldo reflejado del empleado son:

$\begin{array}{ll}- & \text { Aguinaldo } \\ - & \text { Vacaciones y prima vacacional } \\ - & \text { Tiempo extra } \\ - & \text { Día de descanso laborado } \\ - & \text { Día festivo laborado } \\ - & \text { Prima dominical } \\ - & \text { Utilidades }\end{array}$

Y algunos de los factores que pueden ser reflejados como una disminución en la percepción de una nómina son:

\section{Retardos}

Faltas

Fondo de ahorro

Servicio de comedor

\author{
- Habitación \\ - Infonavit \\ - Fonacot \\ - $\quad$ Préstamos otorgados a trabajadores
}

\section{ERP}

Planificación de recursos empresariales. Se refiere a los paquetes de sistemas y software que utilizan las organizaciones para administrar las actividades diarias del negocio, tales como la contabilidad, el abastecimiento, la administración de proyectos y la fabricación. Los sistemas de ERP se unifican, definen una variedad de procesos de negocios y habilitan el flujo de datos entre ellos. Al recopilar los datos transaccionales compartidos por una organización desde diversas fuentes, los sistemas de ERP eliminan la duplicación de datos y garantizan su integridad con una sola "fuente de verdad" [7,8].

\section{Sistemas Informáticos}

Para el desarrollo del módulo de nómina que será adaptado al sistema ERP ya instalado en la empresa, se requerirán las siguientes herramientas de desarrollo.

\section{- $\quad$ Visual Studio (C\#) [9]}

Aplicaciones para dispositivos

Plataforma universal de Windows, iOS y Android

Herramientas de Apache Córdova, Xamarin y Unity pueden descargarse para poder codificar, depurar, probar, compartir y reutilizar en más plataformas.

\section{Herramientas web}

Se crearon e implementaron aplicaciones web modernas

Se desarrolló en web con ASP.NET, Node.js, Python y JavaScript. Se usa con marcos web potentes como AngularJS, jQuery, Bootstrap, Django y Backbone.js.

I

ntegración con Git

Hospedando código en cualquier lugar 
Administrando el código fuente en repositorios Git que hospede cualquier proveedor, incluido GitHub. O bien use Azure DevOps para administrar el código junto con los errores y elementos de trabajo de todo el proyecto.

\section{SQLServer [10]}

Para administrar la base de datos

\section{- $\quad$ Crystal Reports}

SAP Crystal Server, para inicio de sesión único para asegurar y simplificar el acceso de los usuarios con la integración de LDAP y AD.

Seguridad de datos granulares

Gestionar informes

Analítica de negocios móviles.

Habilitar el acceso móvil seguro a los informes de BI de autoservicio [11].

\section{Metodología}

Aplicando la metodología ágil de desarrollo Scrum, a fin de tener una mejor comunicación con el cliente, así como para reducir el periodo de tiempo de creación del sistema [12-14].

Siguiendo las etapas:

$\begin{array}{ll}- & \text { Planeación } \\ - & \text { Diseño } \\ - & \text { Desarrollo } \\ - & \text { Implementación } \\ - & \text { Pruebas }\end{array}$

\section{Planeación}

Levantamiento de requerimientos:

- Comprender el flujo de la información a fin de generar el sistema de nómina.

- Analizar el software ERP ya implementado en la empresa.

- $\quad$ Diseño del sistema

\section{Diseño}

Creación de la interfaz final, considerando un sistema predecible y detallado, que ofrece, una mejor experiencia de usuario. Y la relación entre interfaces.

\section{Desarrollo}

Análisis de la base de datos existente en el sistema ERP de la empresa, con el propósito de adaptar el módulo de nómina requerido. Para tal efecto, se consideró la tabla Usuarios, la cual contiene los datos específicos de todo el personal registrado como trabajador.

Se decide usar las tecnologías (C\#, SQL Server y Crystal Reports), debido a que el sistema ERP con el que ya contaba la empresa, estaba desarrollado en C\# y la base de datos en SQL Server. SQL Server, permite una comunicación totalmente nativa, eficaz y rápida entre C\# (interfaces y lógica) y la base de datos. Además, Crystal Reports es considerada, por ser robusta, amplia y fácil de utilizar.

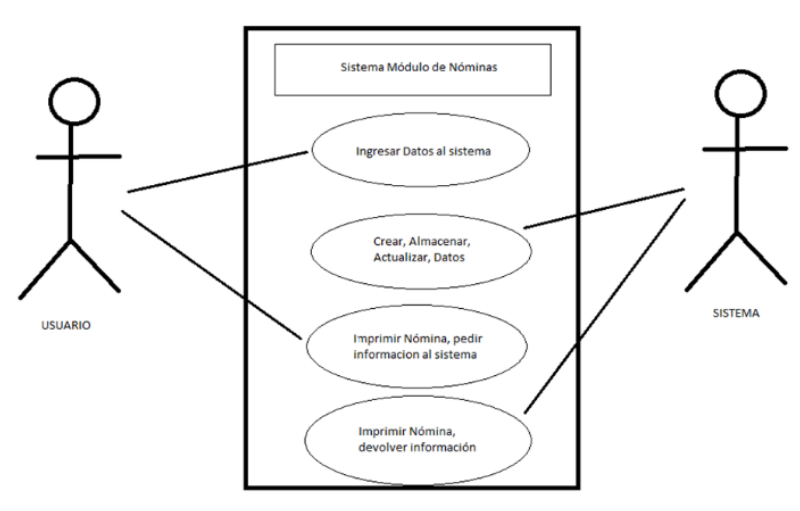

Figura 1 Caso de uso

\section{Resultados}

Se implemento el sistema y se puso en operación a fin de realizar las pruebas requeridas por el usuario. A continuación, se presenta información de los resultados obtenidos. La tabla 1. Muestra el antes y después del módulo.

\begin{tabular}{|ll|l|l|}
\hline \multicolumn{1}{|c}{ Actividad } & \multicolumn{1}{c|}{$\begin{array}{c}\text { Tiempo de } \\
\text { duración } \\
\text { Sin Módulo }\end{array}$} & \multicolumn{1}{c|}{$\begin{array}{c}\text { Tiempo de } \\
\text { duración } \\
\text { Con Módulo }\end{array}$} \\
\hline $\begin{array}{l}\text { Cálculo de } \\
\text { nómina }\end{array}$ & 1.5 Horas & 3 segundos \\
\hline $\begin{array}{l}\text { Creación de } \\
\text { nómina }\end{array}$ & 1.0 Horas & 20 segundos \\
\hline Total & 2.5 Horas & 23 segundos \\
\hline
\end{tabular}

Tabla 1 Comparación de las actividades sin y con módulo de nómina 
Tras la implementación del módulo, en cerca de medio día laboral (en promedio) la actividad es terminada, lo que permite al personal, el realizar más actividades que tengan pendientes, adelantar trabajo y en general, mejorar sus procesos.

Dicho lo anterior, se presentan algunas de las interfaces del Módulo:

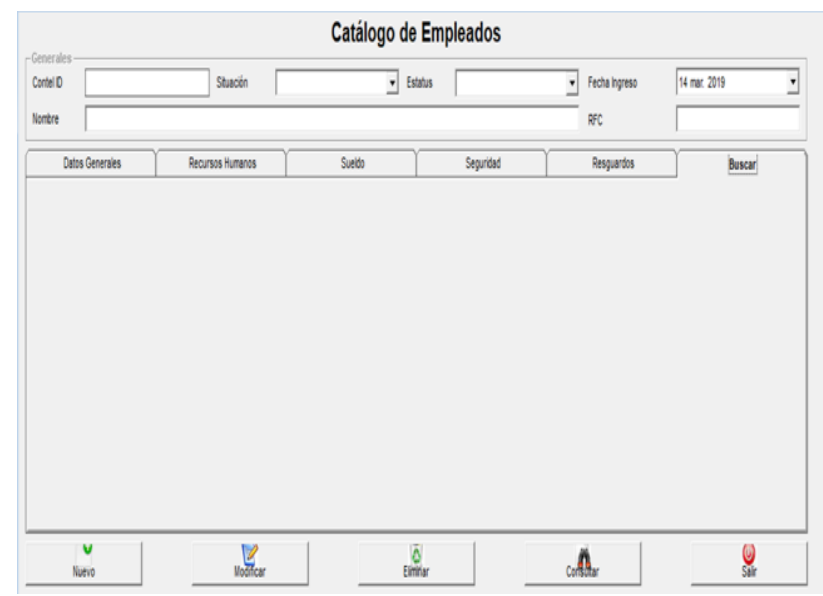

Figura 2 Interfaz principal

La figura 2, muestra el panel principal del módulo implementado, se tiene el título en la parte superior, a continuación se muestra un área con algunos campos vacíos, estos campos son para agilizar la búsqueda de empleados, una vez que se introduce la información la cual puede ser solo el ID, se presiona el botón de "Consultar", el cual cargara toda la información guardada en la base de datos de ese usuario en las pestañas inferiores, así mismo, si se trata de un nuevo usuario, se pueden llenar los datos y hacer clic en "Nuevo", esto creara un nuevo registro y los campos en las pestañas inferiores quedará vacío para ser llenado posteriormente. Entre las pestañas se encuentra:

Datos Generales: En esta sección se incluye los campos a llenar con la información del empleado (véase figura 3).

- Recursos Humanos: Esta sección es para detallar más datos relacionados con algún empleado como lo es, su tipo de contrato, el área, sus horarios, etc; (véase figura 4).

- Sueldo: Esta pestaña contiene todos los datos relacionados con el sueldo que va a percibir cada empleado (ver figura 5).

Seguridad: En esta pestaña se le pueden dar ciertos privilegios a los usuarios, por ejemplo, poder entrar a la empresa con la credencial (Sistema de identificación automatizado)
Resguardos: Fase de diseño, este módulo quedo pendiente, en teoría se trata de que, en algunas ocasiones, a cierto personal se le hace el préstamo de algún equipo, por ejemplo, una computadora, en este apartado se detallará esa situación.

Buscar: Esta pestaña borrará todo lo que se haya buscado con anterioridad (reinicio de la página) y dejará la interfaz como al principio (ver figura 3 ).

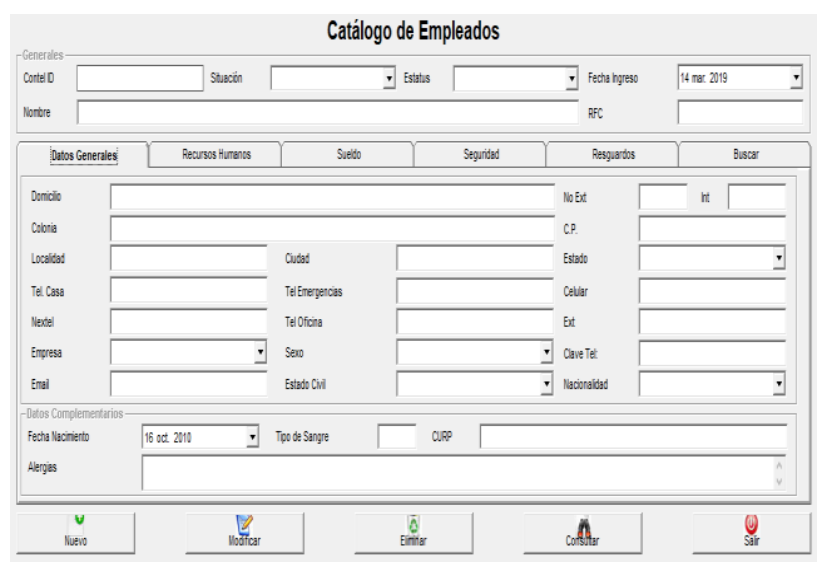

Figura 3 Interfaz "Datos Generales"

Mediante la interfaz presentada en la figura 3, se puede consultar información de un usuario o para ingresar información de alguno nuevo. En este caso aparece como sería el ingreso de nueva información y básicamente eso es todo lo que se ocupa saber de cada empleado.

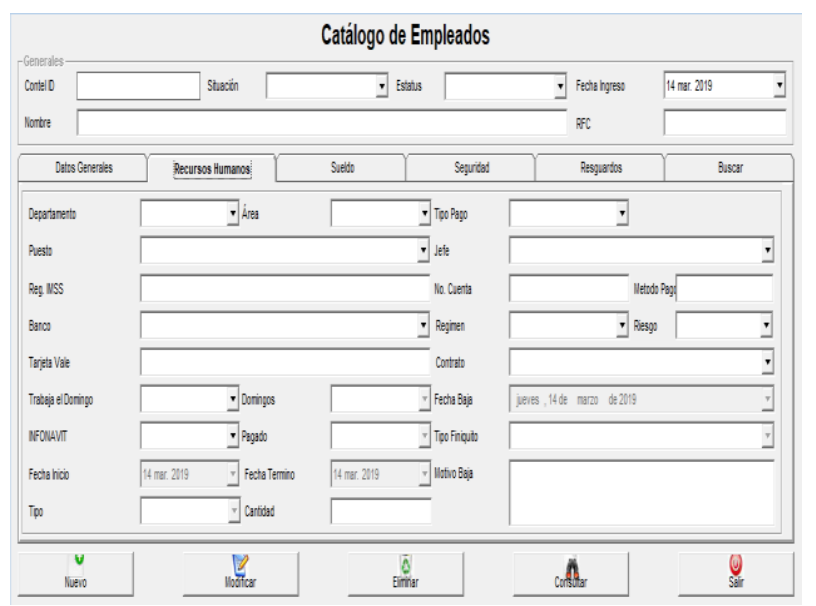

Figura 4 Interfaz "Recursos Humanos"

En la figura 4, se ilustra la interfaz de recursos humanos. Se presenta información específica del trabajador, tal como: su cuenta de banco en la cual se depositará su sueldo, también se designa quien es su jefe inmediato, área de trabajo y fecha de contrato, etc. 


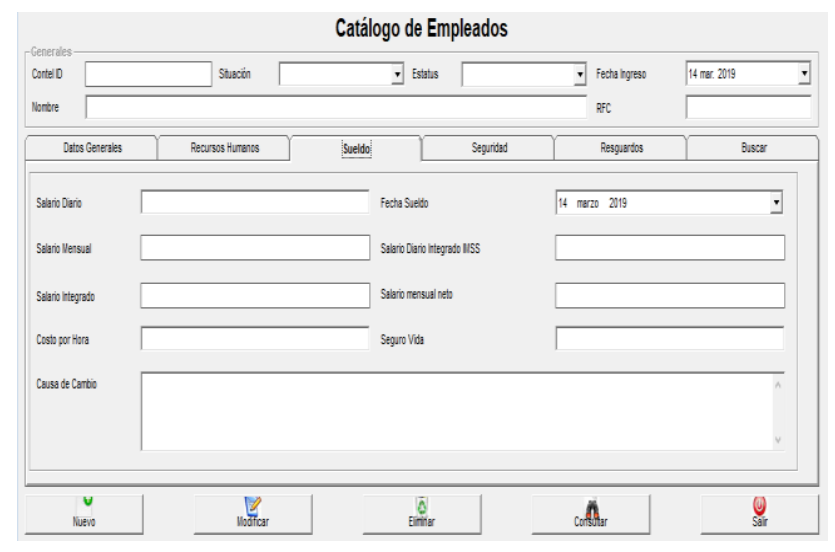

Figura 5 Interfaz "Sueldo"

Finalmente, en la figura 5 se ilustra la interfaz referente al sueldo del empleado. Mostrando datos como: sueldo diario, sueldo mensual, sueldo neto, fecha de pago.

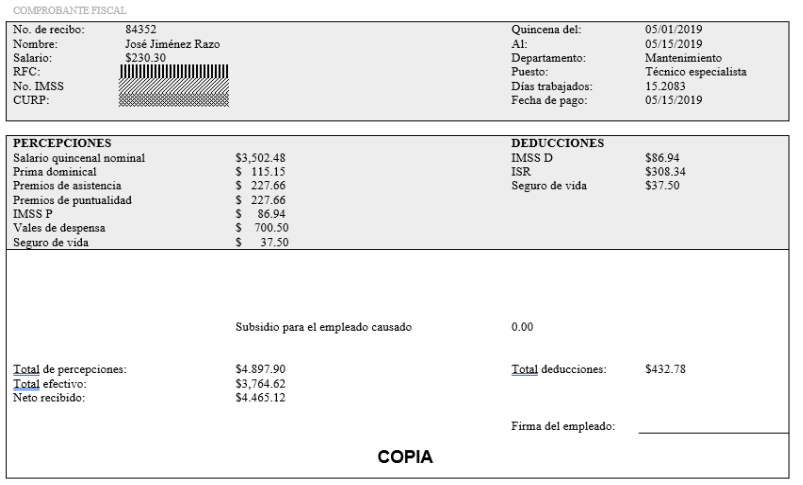

Figura 6 Documento de nómina

Mientras que la figura 6 muestra el documento de nomina como resultado del sistema desarrollado.

\section{Conclusiones}

El módulo de nómina creado y adaptado al software ERP ya implementado en la empresa, trajo consigo una mejora optimizando los procesos. Disminuyendo el tiempo en las operaciones, tal como se aprecia en la tabla 2. Permitiendo al departamento de recursos humanos una mejor administración y control de sus procesos; por lo que, a diferencia de otros como, CONTPAQi Nominas, Aspel-NOI, HR Corporate y Nominasol, este sistema puede ser editado, modificado y adaptado a las necesidades de los procesos que en la empresa se llevan a cabo, especialmente por el sistema que ya manejaba, el cual también fue un desarrollo a la medida.

\section{Referencias}

[1]www.contpaqi.com/nominas/;[02/04/19].

[2]www.sdelsol.com/programa-nominasnominasol/; [02/04/19].

[3]http://fcasua.contad.unam.mx/apuntes /interiores/docs/2005/contaduria/opt/ 2006.pdf *Ese es el link, el 2005 es la fecha y el 2006 es una clave.

[4]www.imss.gob.mx/sites/all/statics/pdf /leyes/LSS.pdf; [22-06- 18].

[5]www.senado.gob.mx/comisiones /desarrollo _social/docs/marco/Ley_FT .pdf; [09-04-

[6]www.imss.gob.mx/patrones/sua; [02/04/17].

[7]Lance Ashdown, Tom Kyte Oracle "Database Concepts", 11g Release 2 (11.2), Mayo 2015.

[8]www.oracle.com/applications /erp /what-iserp.html; [03/04/19].

[9]https://visualstudio.microsoft.com /es/vs/community/; [03/04/19]

[10]www.microsoft.com/en-us/sql-server/sqlserver-2019; [03/04/19]

[11] www.crystalreports.com/; [02/04/19]

[12] O. Salo and P. Abrahamsson, "Agile methods in European embedded software development organisations: a survey on the actual use and usefulness of Extreme Programming and Scrum"; IET Digital Library. Volume 2, Issue 1, February 2008, p. 58 - 64

[13] Kaushal Pathak and Anju Saha, "International Journal of Advanced Research in Computer Science and Software Engineering "'; Kaushal et al., International Journal of Advanced Research in Computer Science and Software Engineering 3(2), February - 2013, pp. 270-276.

[14] www.scrum.org/resources/blog /que-esscrum; [25/09/17]. 